\title{
Deep learning-enabled realistic virtual histology with ultraviolet photoacoustic remote sensing microscopy
}

Matthew Martell

https://orcid.org/0000-0001-9927-894X

Nathaniel Haven

University of Alberta

Ewan McAlister

University of Alberta

Brendon Restall

University of Alberta

Brendyn Cikaluk

University of Alberta

Rohan Mittal

University of Alberta

Benjamin Adam

University of Alberta

Nadia Giannakopoulos

University of Alberta

Lashan Peiris

University of Alberta

Jean Deschenes

University of Alberta

Xingyu Li

University of Alberta

Roger Zemp ( $\nabla$ rzemp@ualberta.ca )

University of Alberta

Article

Keywords:

Posted Date: February 24th, 2022

DOI: https://doi.org/10.21203/rs.3.rs-1377499/v1 
License: (c) (i) This work is licensed under a Creative Commons Attribution 4.0 International License. Read Full License 


\title{
Deep learning-enabled realistic virtual histology with ultraviolet photoacoustic remote sensing microscopy
}

\author{
Matthew T. Martell ${ }^{1, \dagger}$, Nathaniel J.M. Haven ${ }^{1, \dagger}$, Ewan A. McAlister ${ }^{1}$, Brendon S. Restall ${ }^{1}$, \\ Brendyn D. Cikaluk ${ }^{1}$, Rohan Mittal ${ }^{2}$, Benjamin A. Adam ${ }^{2}$, Nadia Giannakopoulos ${ }^{2}$, Lashan \\ Peiris $^{3}$, Jean Deschenes ${ }^{2}$, Xingyu $\mathrm{Li}^{1}$, and Roger J. Zemp ${ }^{1, *}$ \\ ${ }^{1}$ Department of Electrical and Computer Engineering, University of Alberta, 116 Street \& 85 Avenue, Edmonton, \\ Alberta T6G 2R3, Canada \\ ${ }^{2}$ Department of Laboratory Medicine and Pathology, University of Alberta, 8440 - 112 Street, Edmonton, Alberta \\ T6G 2B7, Canada \\ ${ }^{3}$ Department of Surgery, University of Alberta, 8440 - 112 Street, Edmonton, Alberta T6G 2B7, Canada \\ *rzemp@ualberta.ca \\ †These authors contributed equally to this work.
}

\begin{abstract}
The goal of oncologic surgeries is to completely resect tumor tissue, yet in up to $40 \%$ of such surgeries, positive margins are found in the resected tissues. Postoperative histology using H\&E-stained brightfield microscopy is the gold standard for determining margin status, but rapid frozen section analysis is sometimes performed for intraoperative guidance, albeit with inaccuracies. In this work, we introduce a virtual histological imaging method based on a non-contact, reflection-mode ultraviolet photoacoustic remote sensing and scattering microscope, combined with deep learning using a cycle-consistent generative adversarial network. The system is capable of high-resolution scanning with $390 \mathrm{~nm}$ resolution comparable to conventional histopathology, and fast widefield scanning, generating images with histological realism in freshly-resected thick tissues or thin sections. Cytologic and architectural features of interest are readily identifiable in virtual histology images of benign and malignant tissues. To evaluate system performance, a blinded study of pathologists rating image quality metrics was conducted, with our virtual histology approach offering preferred hematoxylin-like detail $(P=0.0018)$ and overall stain quality $(P=0.0321)$ compared to frozen section analysis.
\end{abstract}

\section{Introduction}

Surgical resection is the primary treatment for many solid tumors. In oncologic surgeries, surgeons attempt to excise the malignant tissue along with a surrounding margin of normal tissue, while sparing as much healthy tissue as possible. The margins of a resected specimen are then evaluated for involvement of malignant cells, ensuring that the primary cancer has been completely removed. The status of surgical margins is therefore considered one of the strongest indicators of operative success, and long-term patient prognosis. Unfortunately, gross visual inspection of resection margins offers limited sensitivity for delineating boundaries between healthy and malignant tissues, allowing microscopic pathology to go undetected. Positive margins are still found under postoperative analysis in up to $40 \%$ of cases ${ }^{1}$ depending on the type of tumor, often necessitating follow-up re-excision surgery, and adjuvant chemotherapy, radiation, or endocrine therapies. The problem of positive margins not only causes additional physical and emotional trauma for patients and leads to increased morbidity, but also results in delays, excess expenses, and consumption of limited resources within the overall healthcare system ${ }^{2}$. The development of improved microscopic imaging methods for intraoperative margin status assessment could significantly enhance patient care and contribute to a more cost-efficient healthcare economy.

The current gold standard for margin assessment involves pathologist inspection of hematoxylin and eosin (H\&E) stained thin sections of tissues under brightfield microscopy. This allows identification of atypical cytologic features including an increased nucleus-to-cytoplasm ratio, variations in the size and shape of cells and nuclei, and increased or abnormal mitosis, in addition to various architectural patterns which differentiate benign and malignant tissues. However, preparation of formalin-fixed paraffin-embedded (FFPE) tissue sections compatible with transmission-mode light microscopy involves many time-consuming steps (Fig. 1a), typically resulting in minimum turnaround times of nearly 24 hours. FFPE H\&E histopathology is therefore unsuitable for providing real-time intraoperative feedback.

Conventional alternatives to FFPE H\&E histopathology exist to offer rapid assessment of margin status while the patient remains in the surgical suite, though they suffer from limitations in technical quality and diagnostic accuracy in some 


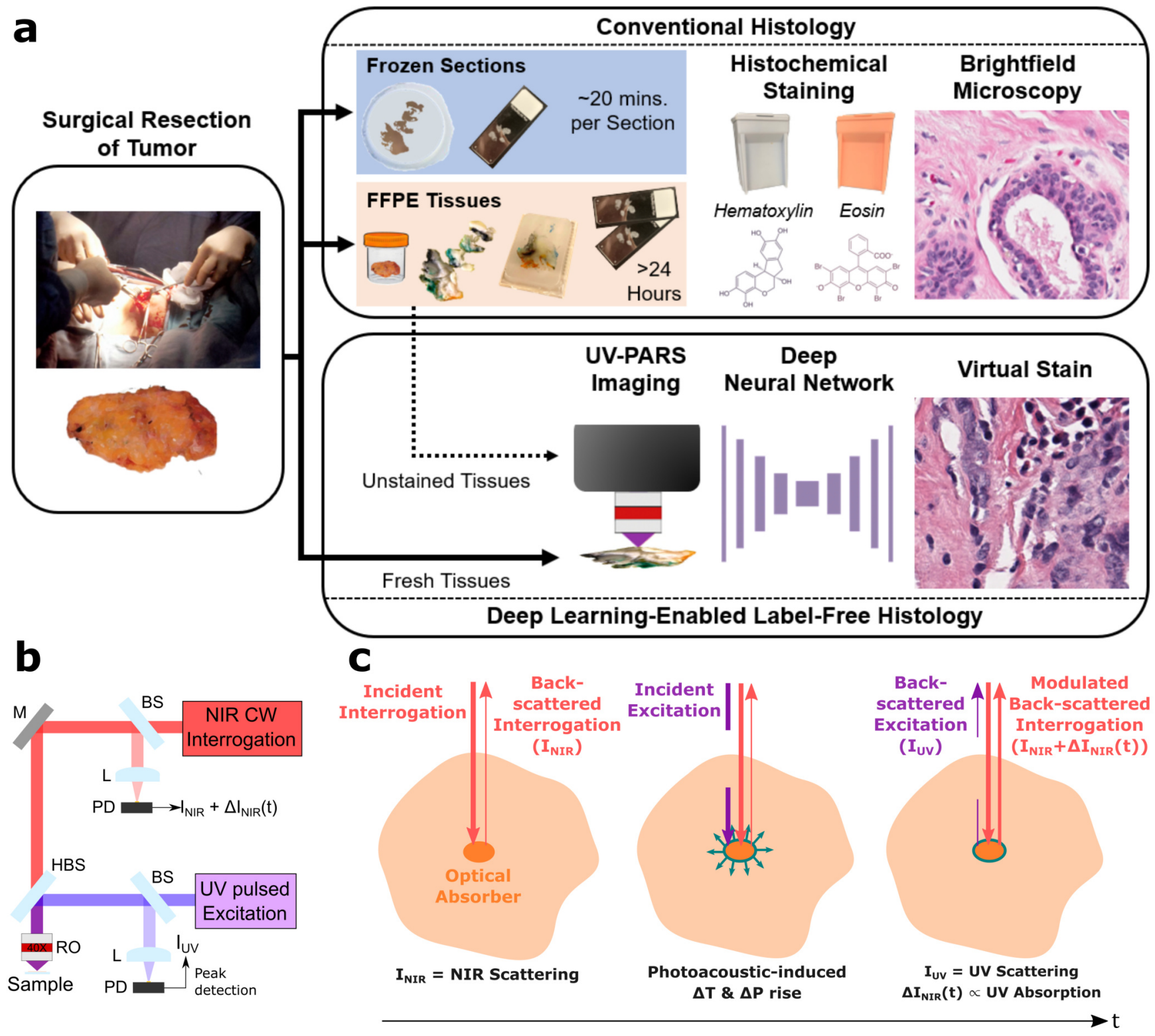

Figure 1. a) Conventional surgery-to-histopathology workflow (top) comparing frozen sections to FFPE tissue methodologies, in contrast to our deep learning-enabled label-free histology approach (bottom) that is capable of bypassing tissue preparation and/or staining steps to rapidly obtain virtual histology. b) A simplified system diagram of our combined ultraviolet photoacoustic remote sensing and ultraviolet scattering microscopy system capable of providing simultaneous absorption and scattering contrast, respectively. M, mirror; L, lens; BS, beamsplitter; HBS, harmonic beamsplitter; RO, reflective objective; PD, photodiode. c) Visualization of the photoacoustic remote sensing mechanism. 
applications. Frozen section analysis (FSA) can generate slides available for brightfield microscopy in approximately 20 minutes. However, drawbacks include errors due to limited sampling of the tissue, a high dependence on operator skill, and the destructive nature of the tissue preparation process, which may leave insufficient tissue for subsequent permanent sections to provide a definitive diagnosis. Frozen sections are also subject to significant freezing artifacts, which lead to diagnostic inaccuracies ${ }^{3}$. Moreover, lipid-rich tissues such as the breast do not freeze well and are difficult to cut into thin sections. FSA is consequently not performed routinely in resection surgeries for the majority of tumor types.

Recent efforts have developed methods capable of virtual histological imaging. These methods aim to provide histological feedback early in the standard histopathology workflow (Fig. 1a), but advantages over FSA have yet to be established. In this study, we introduce a multilayer, high-resolution virtual histology method which achieves histological realism in unstained tissues and demonstrates advantages over FSA in a blinded pathologist study. In brief, our approach uses ultraviolet photoacoustic remote sensing (UV-PARS) microscopy to achieve virtual hematoxylin contrast to visualize cell nuclei, and ultraviolet scattering microscopy to provide virtual eosin contrast for imaging cytoplasm and the extracellular matrix (Fig. 1b). PARS is a non-contact laser scanning photoacoustic imaging modality which detects intensity modulations in a back-scattered interrogation beam induced by optical absorption of a nanosecond-pulsed excitation beam ${ }^{4,5}$ as shown in Fig. 1c. UV-PARS was first demonstrated by Haven et al. ${ }^{6}$ for cell nuclei imaging and further explored in other reports ${ }^{7,8}$. Near-infrared (NIR) scattering microscopy was subsequently integrated to provide complementary virtual eosin contrast ${ }^{9,10}$, which was previously obtained using more complex approaches ${ }^{11,12}$. The NIR scattering was later upgraded to use $266 \mathrm{~nm}$ pulsed UV scattering along with a pulse peak sample-and-hold detection circuit to eliminate the discrepancy in lateral resolutions between the hematoxylin-like and eosin-like contrast ${ }^{13}$. In this work we use two approaches for scanning (see Figure 1 in the supplementary information): (1) a high-resolution 0.5 numerical aperture (NA) objective stage-scanning mode capable of imaging $1 \mathrm{~mm}^{2}$ in several minutes with $390 \mathrm{~nm}$ resolution, and (2) a rapid lower-resolution mode using a long working distance 0.3 NA objective with a hybrid optomechanical scanning mechanism capable of imaging $1 \mathrm{~mm}^{2}$ in $4.2 \mathrm{~s}$ with $860 \mathrm{~nm}^{\text {resolution }}{ }^{14}$.

Compared to previous techniques such as multiphoton ${ }^{15}$ and confocal fluorescence microscopy ${ }^{16}$, light-sheet microscopy ${ }^{17}$, microscopy with ultraviolet surface excitation (MUSE) ${ }^{18}$ and related methods, our approach is entirely label-free. In contrast to ultraviolet photoacoustic microscopy (UV-PAM) ${ }^{19-25}$, another label-free approach, our method achieves higher spatial resolution and is non-contact, requiring no acoustic coupling medium. Our all-optical, reflection-mode setup additionally simplifies imaging of thick samples. SLAM microscopy is another label-free method which offers multiple simultaneous endogenous contrasts highlighting structural and metabolic factors in the tumor microenvironment ${ }^{26}$. However, it was not able to generate images with positive contrast for cell nuclei like traditional histopathology. Moreover, stimulated Raman scattering (SRS) microscopy has generated impressive images, though they currently lack the histological realism of brightfield microscopy ${ }^{27}$ and are subject to slower scan times $\left(25 \mathrm{~s} / \mathrm{mm}^{2}\right)^{28}$. Our approach is capable of fast image acquisition $\left(4.2 \mathrm{~s} / \mathrm{mm}^{2}\right)$ with sufficient histological realism and image quality preferred over FSA.

To render our virtual histological images with maximal realism comparable to true H\&E-stained brightfield microscopy, we leverage deep learning using a cycle-consistent generative adversarial network (CycleGAN) ${ }^{29}$ approach. A generative adversarial network (GAN) in general uses the concept of adversarial training, where a generator network generates new candidate data in a target domain, and is trained in zero-sum competition with a discriminator network which aims to differentiate real input example data from the fake generator outputs ${ }^{30}$. However, in the context of image-to-image translation, more traditional generative adversarial networks like the conditional GAN (cGAN) ${ }^{31}$ approach condition the GAN model on the input of class labels, or notably on input images in the case of the well-established pix 2 pix model $^{32}$. This requires supervised training with one-to-one paired input data. CycleGANs can use unpaired training data to learn the image-to-image transformation between two domains in an unsupervised manner. Cycle consistency constraints ensure that transformation to another domain and back achieves an image as close as possible to the starting image. This is analogous to a comment attributed to Mark Twain that if one translates a sentence from English to French and back, one should arrive at the same starting sentence. In a mathematical sense, the cycle consistency constraint approximately imposes a mutual inverse relationship between two generative models trained simultaneously for a forward and reverse transformation. This serves to regularize the one-to-many problem of adversarial training of the forward transformation with unpaired data to a one-to-one mapping, given that functions are invertible if and only if they possess the property of bijectivity. In our situation we consider virtual histology and true H\&E brightfield microscopy image domains, $X$ and $Y$, respectively (Fig. 2b). We use these images as unpaired inputs into a CycleGAN algorithm. As shown in Fig. 2b, the CycleGAN learns the generator network transformation $G$ between the virtual and real H\&E image domain as well as the inverse transformation $F$ between real and virtual domains, then imposes cycle-consistency constraints to ensure maximal realism as governed by discriminator networks $D_{Y}$ and $D_{X}$ classifying translated images as real or fake. The detailed architectures of these deep convolutional neural networks are outlined in Fig. 2c. The CycleGAN approach is important in our context because it is not always possible to achieve virtual histology images with a paired true H\&E counterpart. This is particularly true for thick tissues and multi-layer imaging, where the fixing, embedding, sectioning and staining processes may create significant registration errors. An additional advantage of 
the CycleGAN approach is that we can achieve stain-style matching to existing digital histology databases, without requiring an entirely new training set of paired example images in the target stain style. This will be important for minimizing stain variability, and for applying computer-aided diagnosis algorithms for digital pathology to our virtual histology images in future work. The unpaired training of the CycleGAN model may additionally facilitate integration into existing clinical workflows, as the output virtual H\&E stain style can be targeted to match individual pathologist preferences or standardized institutional protocols, requiring only one new set of true stained images rather than paired examples.

To generate virtually-stained images emulating brightfield H\&E-stained microscopy, we first combine UV-PARS and UV scattering data to form pseudo-colored virtual histology images as shown in Fig. 2a. This is performed by a custom image fusion method ${ }^{13}$ based on a reference H\&E-stained brightfield microscopy image, along with a blind source stain separation algorithm ${ }^{33,34}$. These initial images approximating true histology are used as inputs to the trained CycleGAN algorithm. In this report, we test our approach on formalin-fixed breast and prostate tissues from human subjects as well as on freshly-resected murine liver and kidney tissues, including optically-sectioned sub-surface imaging.

Recent work with deep learning and autofluorescence ${ }^{35}$ or quantitative phase-contrast microscopy ${ }^{36}$ required thin sections but were not demonstrated in thick tissue. In contrast, our approach is suitable for thick tissue reflection-mode imaging. Generative adversarial networks have additionally been used to achieve virtual images in the style of true H\&E brightfield images in concert with imaging technologies including $\mathrm{MUSE}^{37}, \mathrm{UV}^{-\mathrm{PAM}^{25}}{ }^{25}$, optical coherence tomography (OCT) ${ }^{38}$, and reflectance confocal microscopy $(\mathrm{RCM})^{39}$. 3D OCT and a conditional GAN were used to produce cross-sectional virtual H\&E imagery in unstained thick tissues ${ }^{38}$. However, the spatial resolution of OCT is typically around 20 times coarser than what is currently accepted by pathologists for accurate histological analysis, and hematoxylin-like imaging inferred from the OCT optical scattering contrast with speckle noise is not yet well validated. Conditional GANs were also recently used to transform reflectance confocal microscopy images to a virtual histology rendering ${ }^{39}$. However, this modality used $830 \mathrm{~nm}$ light and only achieved coarse micrometer-scale resolution as input to the cGAN. Additionally, it relies on negative but not positive nuclei contrast in unstained tissues as the cGAN input. As a result, they report only $\sim 80 \%$ sensitivity and $\sim 70 \%$ precision for nuclei prediction on their ex vivo testing image set. This is encouraging, but likely insufficient for confident histopathological diagnosis. In contrast, our combined PARS and scattering data achieves fine $390 \mathrm{~nm}$ resolution, positive contrast for cell nuclei, and histological detail that already closely approximates true H\&E thin sections as input for deep learning methods. Compared to methods relying on autofluorescence for virtual eosin contrast, our UV scattering approach can provides higher signal-to-noise. To our knowledge, the UV-PARS and UV scattering approach is also the first to achieve needed resolution with multi-layer capabilities, and generate sufficient histological detail and fidelity to be preferred over frozen sections. Moreover, our method can achieve fast scanning important for imaging tissues during surgeries with, the long-term goal of obtaining improved surgical outcomes.

To compare stain quality of our virtual histology images against frozen section H\&E-stained images, we performed a blinded pathologist study on a sample of human breast tissues. Pathologists were able to identify important histopathological features, and differentiate benign tissues from malignant disease. Based on stain quality metrics, our virtual histology images offered superior nuclear detail and were preferred overall compared to frozen section H\&E-stained images. Given that our approach can scan large en-face thick tissues within intraoperative time frames, our approach offers promise as an alternative to FSA.

\section{Results}

\section{Virtual staining of breast lumpectomy specimens}

To test our virtual histology approach on human breast tissues, we input pseudo-colored virtual images of sectioned and unstained lumpectomy specimens, unseen in the initial training set, into the CycleGAN and compared results to true adjacent section H\&E brightfield microscopy for validation (Fig. 3). These samples were scanned using the high-resolution mode. Fig. 3a shows benign, moderately dense fibrous stromal tissue with scattered fibroblasts and lymphocytes. Comparing the true H\&E image shown in (i) to the virtual histology image shown in (ii), the stromal structure and nuclear details are concordant. The virtual staining closely models the characteristic H\&E coloration and also accurately emulates the translucent quality of brightfield microscopy, resulting in realistic images comparable to those which pathologists are accustomed and highly-trained to interpet. Fig. 3b shows another concordant comparison between virtual and adjacent true H\&E images, allowing the striated texture of the eosin stain to be appreciated. Fig. 3c shows a benign duct with surrounding chronic inflammation, with the comparative brightfield H\&E-stained image providing further evidence of the concordance of our method with a true H\&E stain.

Fig. 4 shows several representative deep learning-enabled virtual images provided to three pathologists for annotation. The image quality and the histological features of interest resolved in our images of unstained tissues were sufficient to identify regions of concern for malignancy and invasive disease, or favor a benign interpretation. Invasive carcinoma was readily 

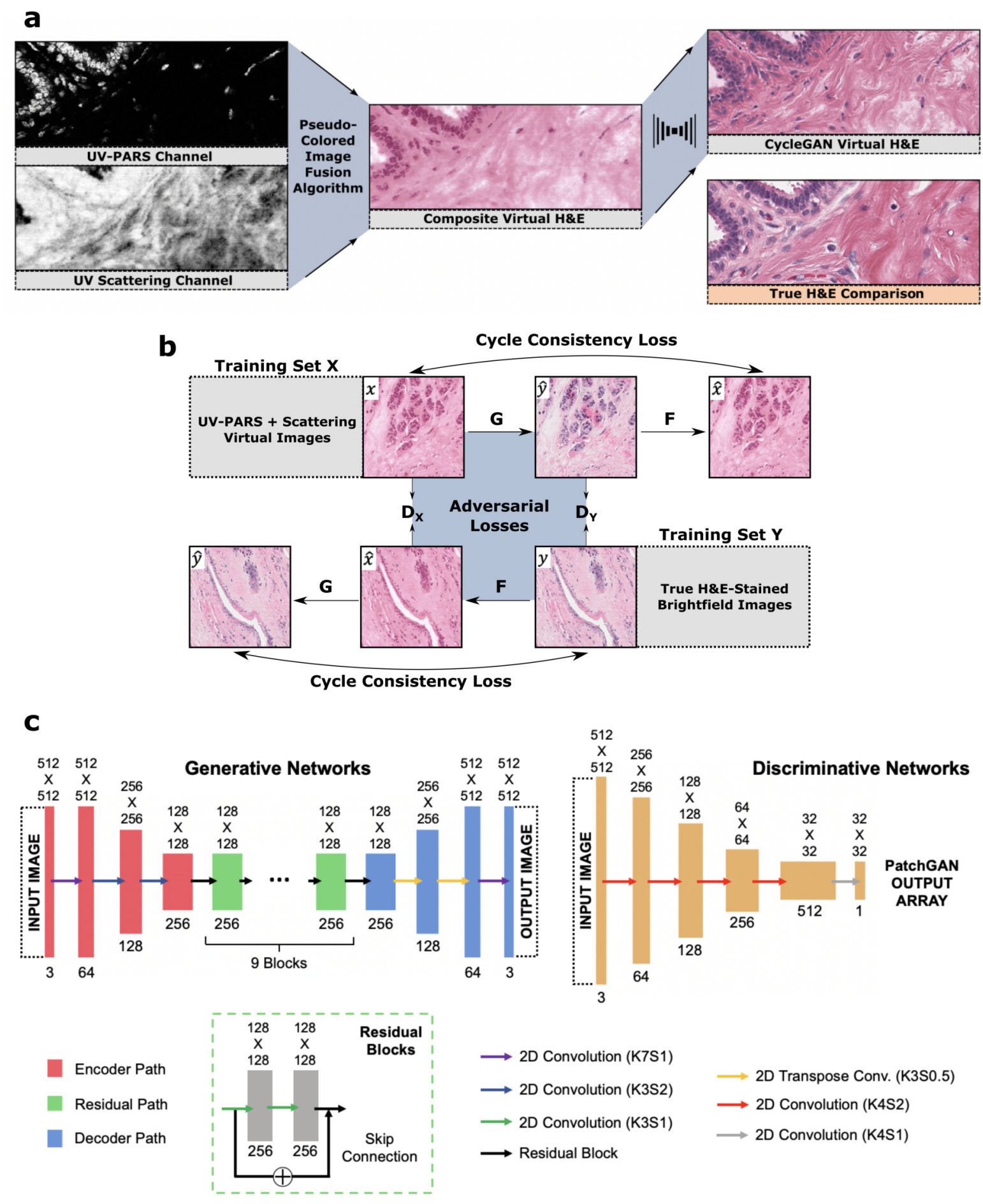

Figure 2. a) Diagram of image acquisition and processing for our virtual histology method. Simultaneously-acquired UV-PARS and UV scattering raw images are combined into a a pseudo-colored intermediate image approximating H\&E histology using a custom image fusion algorithm. These images are input into a trained CycleGAN model for translation into a realistic rendering closely comparable to the style of true H\&E-stained brightfield microscopy. b) Outline of CycleGAN training with unpaired input data sets including virtual images $X$ and true brightfield H\&E-stained images $Y$. Forward and reverse generator translations $G$ and $F$ are trained concurrently with corresponding discriminators $D_{Y}$ and $D_{X}$, which progressively improve their ability to classify generated examples from true input data. Image comparisons used for cycle consistency and adversarial losses are indicated. c) Detailed architectures of the consistuent deep convolutional neural networks used in the CycleGAN model. 

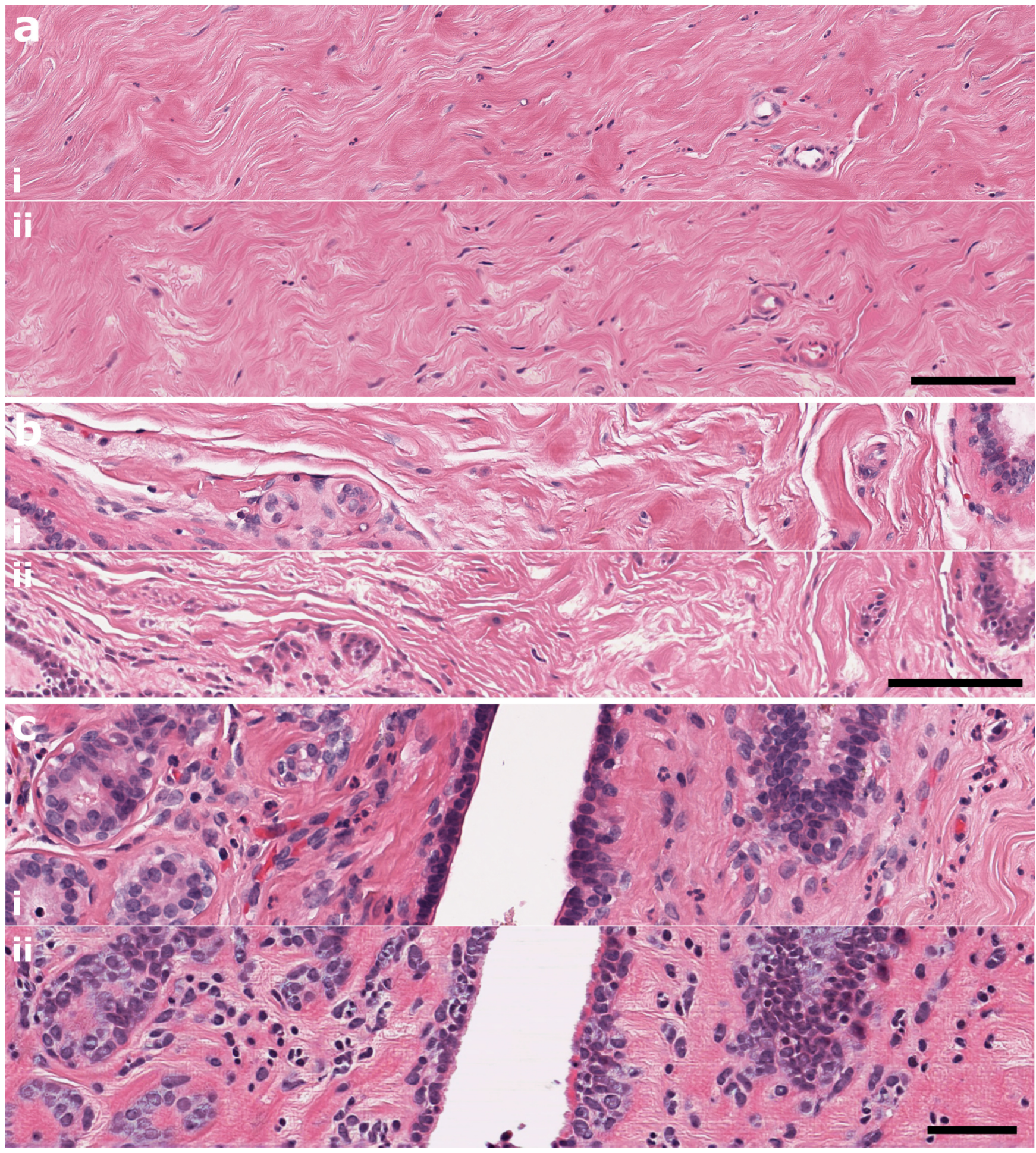

Figure 3. a) and b) Comparisons of deep learning-enabled virtual histology images (ii) with adjacent section true H\&E-stained brightfield images (i) for benign stromal breast tissue. Scale bars: $100 \mu \mathrm{m}$. (c) A similar comparison in breast tissue showing a benign duct with surrounding chronic inflammation. Scale bar: $50 \mu \mathrm{m}$. 
identified in these images, in agreement with the blinded pathology reports associated with these lumpectomy specimens. These reports were signed-out by a different pathologist from those surveyed to interpret and annotate the virtual images.

Pathologist annotation of our virtual images also showcased the range of readily identifiable features which are important for diagnosis. Fig. 5 displays a selected variety of these features appreciated in both benign and malignant tissues. Importantly, pathologists were able to discriminate benign and malignant cytologic features, evaluate tissue architecture, and describe patterns that differentiate invasive malignancy from ductal carcinoma in-situ (DCIS). Notably, as illustrated in Fig. 5 virtual histological quality is sufficient to allow pathologists to recognize and contemplate potentially ambiguous presentations such as a focus of lymphovascular invasion versus a commonly seen retraction artifact, as they would in conventional brightfield $\mathrm{H} \& \mathrm{E}$ histopathology.

\section{Virtual staining of radical prostatectomy specimens}

To test our deep learning-enabled virtual histology approach using the fast-scanning mode, we imaged radical prostatectomy specimens. A mosaic approach was used to stitch individual scan strips to provide a wide field of view, allowing the complex glandular architecture of prostate specimens to be visualized within intraoperative time frames. Due to this scanning method, discrete horizontal swaths are visible due to the mosaic boundaries. However, these do not appear to significantly affect overall image quality and pathologist interpretation. We note in Fig. 6c (ii) that air bubbles found between the tissue surface and coverslip were revealed as artifacts in the composite UV-PARS and UV scattering microscopy pseudo-colored images. Such artifacts are preserved under the CycleGAN translation, despite not being presented as examples during training. This offers evidence that the input morphology is preserved under the translation, which is intended only for style transfer. Comparing our virtual image shown in Fig. $6 \mathrm{~b}$ (ii) to the true H\&E image in (i), there is strong concordance in gross morphology between the two images, with the Fig. $6 \mathrm{~b}$ zoomed inset allowing appreciation of the cell nuclei distribution in glandular benign prostatic hyperplasia. From these images, pathologists were able to describe salient histological features including undulating gland lumens, and cells with a low nucleus-to-cytoplasm ratio favoring a benign interpretation.

\section{Virtual staining of freshly-resected thick murine tissues}

In order to test the capability of this approach for imaging fresh, unprocessed thick tissues, a resected mouse kidney was imaged to generate a z-stack of en-face images in depth as shown in Fig. 7a. This demonstrates the ability of the UV-PARS and UV scattering microscopy system to generate optically-sectioned multi-layer images. Note that direct morphological comparison to brightfield H\&E histology is impractical given the difficulty in precisely maintaining tissue orientation through FFPE tissue processing, which is also known to introduce changes in morphology. However, Fig. 7b shows a zoomed-in comparison of deep learning-enabled virtual H\&E stains in both liver and kidney tissue images to comparable brightfield true H\&E-stained thin tissue sections, illustrating the realism of the virtual stain even with images taken below the surface of thick, unsectioned tissues. Validation of multi-layer UV-PARS imaging has been previously confirmed with co-scanned UV-PARS and confocal fluorescence microscopy ${ }^{9}$. Results additionally appear to support the success of the transfer learning approach in matching the virtual histological images to their true H\&E-stained counterparts.

\section{Blind evaluation of virtual staining approach}

To evaluate the performance of our imaging approach, we compared deep learning-enabled virtual histology images to H\&Estained frozen sections, the current rapid intraoperative histology method in many potential use cases of our microscopy system. Representative examples of frozen section H\&E-stained breast histology images are shown in Fig. 8c. A blinded pathologist study was conducted, where three pathologists were asked to rank image quality on metrics including hematoxylin detail, eosin detail, and overall stain quality on a scale of 1 to 4 , with scores outlined in Table 1 of the supplementary information. Fig. 8a shows a summary of the mean scores provided by each pathologist for each of the image quality metrics. A sample size of $n=15$ was used for each histological method. In comparing the mean scores between virtual and frozen section H\&E-stained histology, the individual pathologists typically preferred the virtual stain approach in all but one result, where one pathologist rated the frozen section eosin stain detail higher on average. It is worth noting that mean scores for virtual histology all exceed a threshold of 2 which was defined on the rating scale as acceptable stain quality, while the frozen section H\&E-stained images failed to meet this standard. In addition, to mitigate the effect of inter-rater variability, an inter-pathologist average score was computed for each individual image and image quality metric. The means of these scores over all images for the $n=15$ sample of each type are summarized in Fig. 8b, which suggests an overall preference for virtual histological imaging. Moreover, p-values were determined using a right-tailed Wilcoxon rank sum test to compare the sample of images of each histological method across each image quality metric, with the null hypothesis taken as equal median scores for virtual staining and frozen sections. Hematoxylin detail and overall stain quality tests resulted in p-values of 0.0018 and 0.0321 , respectively, supporting the rejection of the null hypothesis at the $95 \%$ confidence level. This suggests a significant preference for virtual images with respect to these metrics. Although the mean eosin detail score for our virtual histology was higher than that for frozen section $\mathrm{H} \& \mathrm{E}-$ stained histology, the significance of this result was not established at the $\alpha=0.05$ level. Overall, the study results 


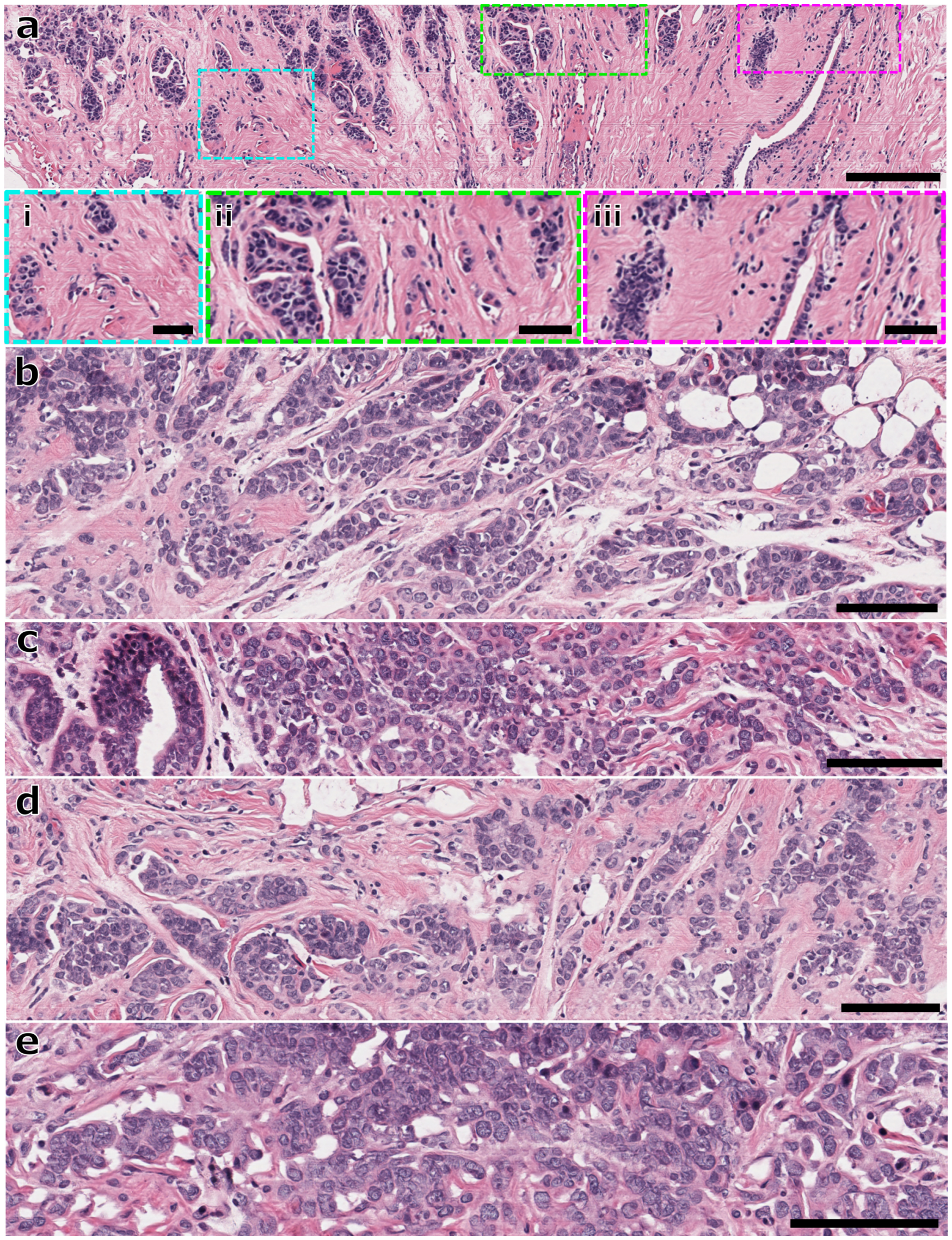

Figure 4. Representative deep learning-enabled virtual histology images of malignant breast tissues, with cytologic details and architectural patterns indicating invasive carcinoma in all examples. Scale bars: a) $200 \mu \mathrm{m}$, (i-iii) $50 \mu \mathrm{m}$, and b)-e) $100 \mu \mathrm{m}$. 


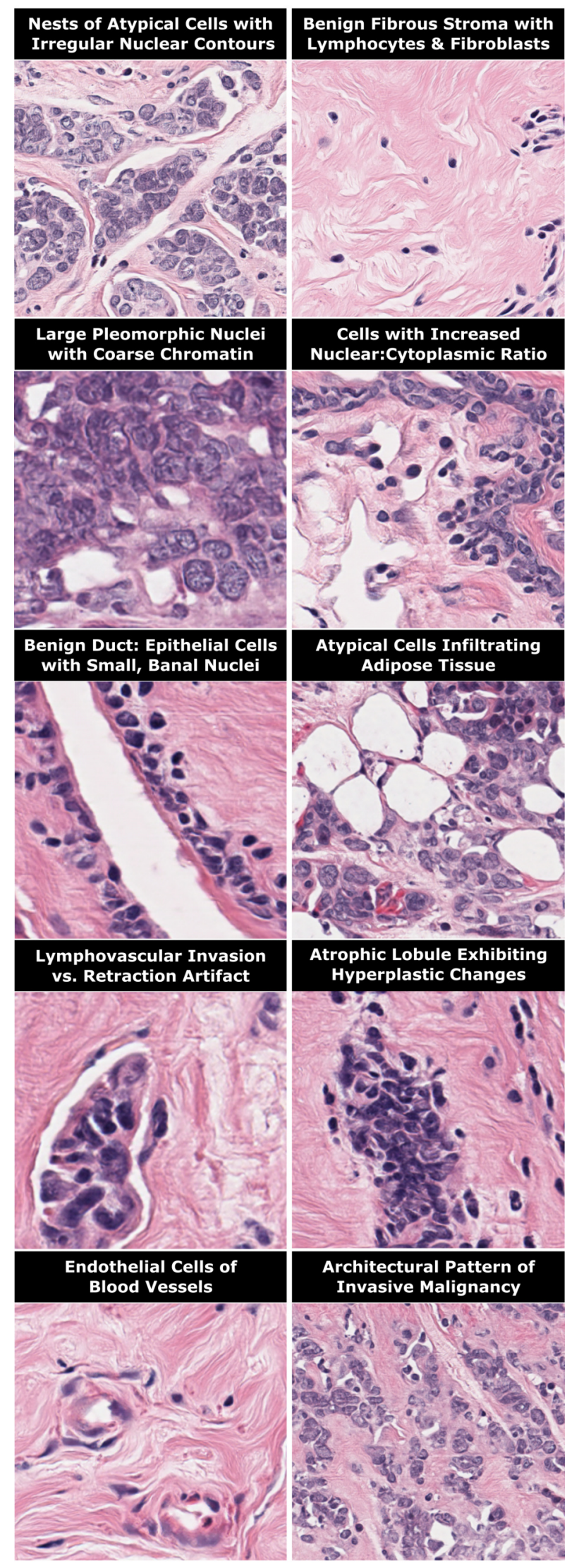

Figure 5. Deep learning-enabled virtual histology images of benign and malignant breast tissues. The system allows pathologists to appreciate various features of diagnostic interest in unstained tissues, as shown in these representative examples. 


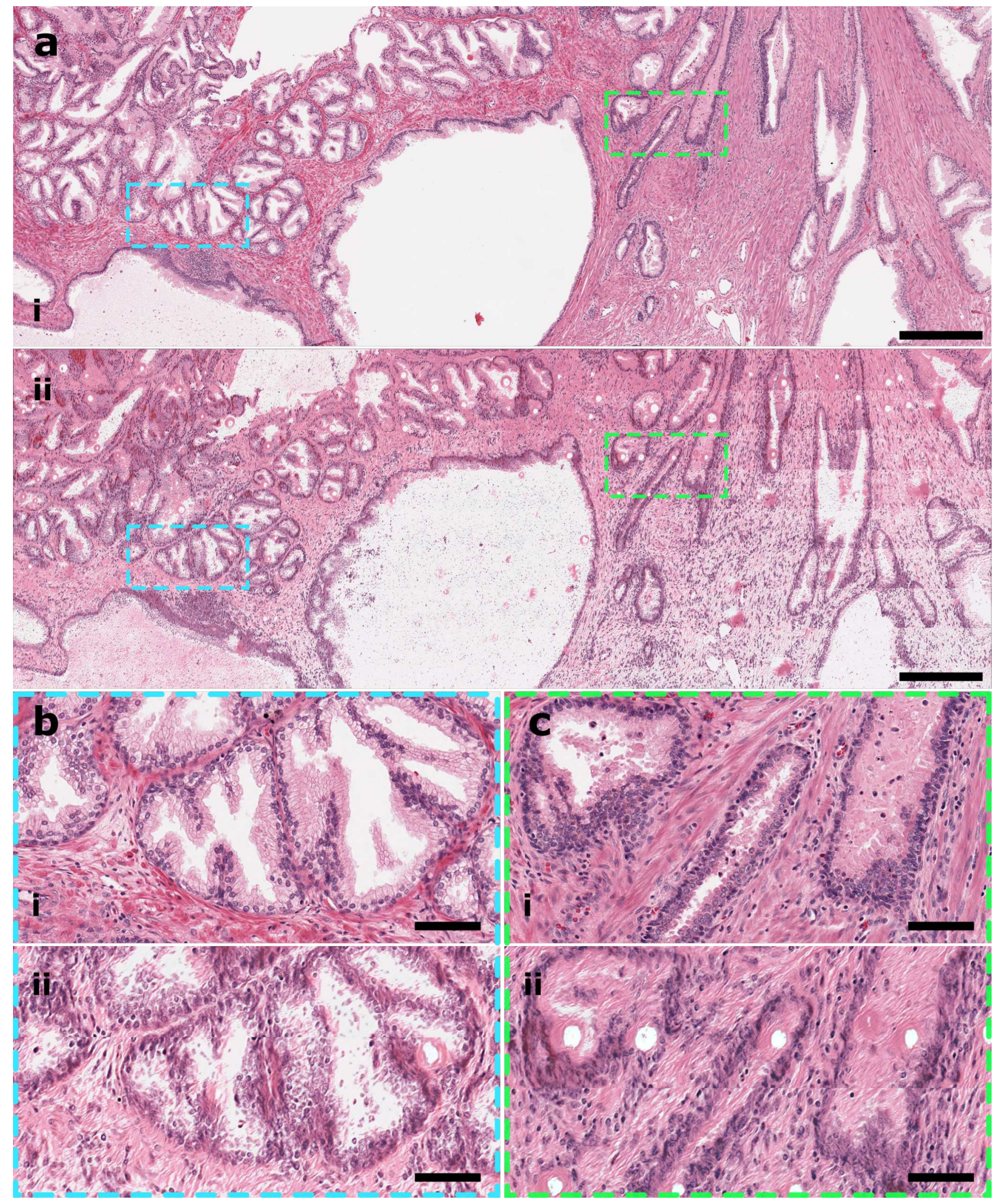

Figure 6. a) A high-speed optomechanical scanning mode deep learning-enabled virtual histology image (ii) compared with an adjacent true H\&E-stained thin section image (i) of human prostate from a radical prostatectomy specimen. Scale bar: 500 $\mu \mathrm{m}$. b-c) Zoomed-in insets showcasing histological detail at higher magnification. Scale bar: $100 \mu \mathrm{m}$. 

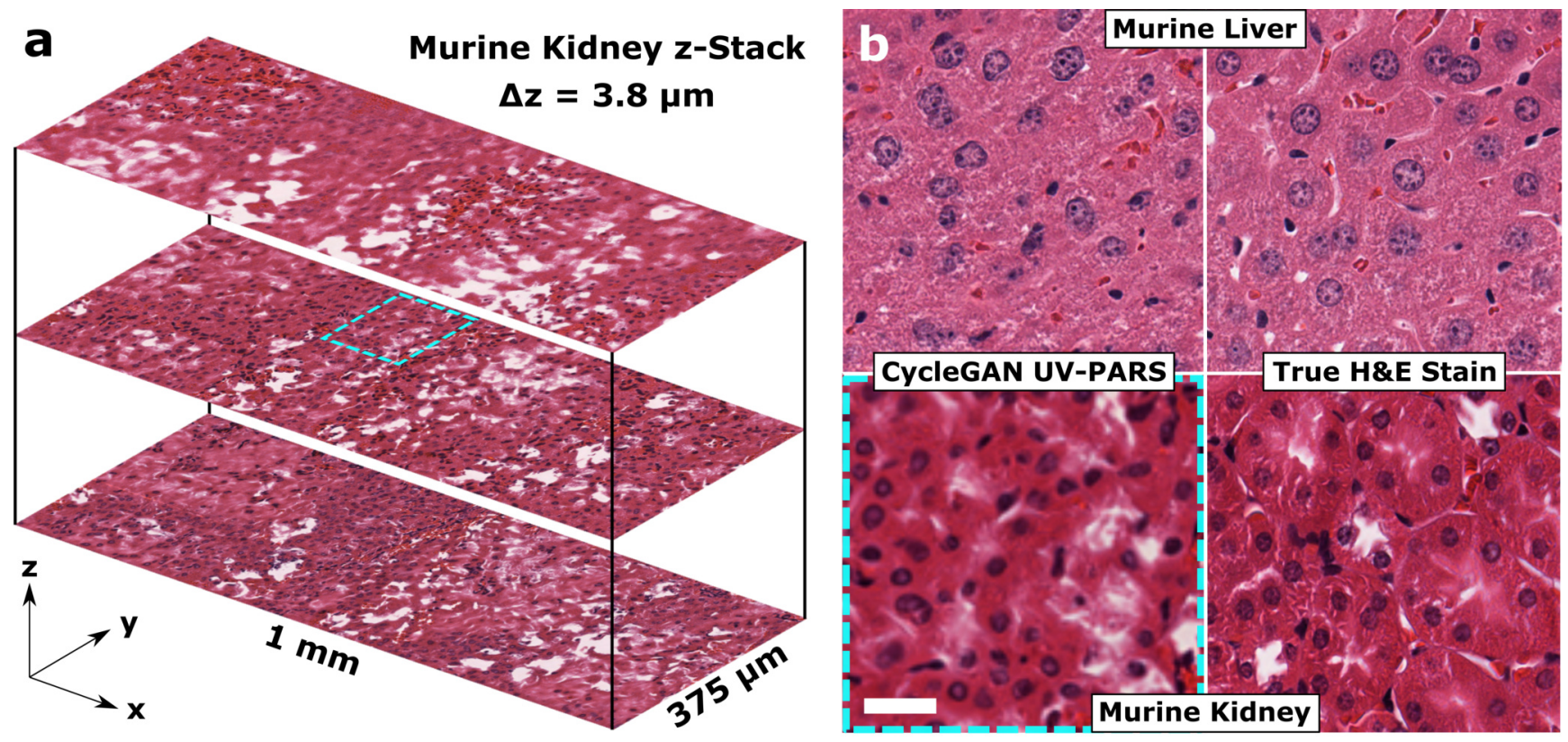

Figure 7. a) Multi-layer z-stack of deep learning-enabled virtual histology images of freshly-resected thick murine kidney tissues, with a representative zoomed-in inset shown in b) highlighted in the dashed box. Unpaired H\&E-stained thin section brightfield images are shown for style comparison with virtual histology results in murine kidney and liver tissues. Scale bar: $25 \mu \mathrm{m}$.

suggest that our deep learning-enabled virtual histology technique is preferred by pathologists over frozen section H\&E-stained histology.

\section{Discussion}

Our imaging approach offers promising results with multi-layered virtual histology capabilities and close concordance with true H\&E-stained histology. The success of this method can be attributed substantially to achieving positive contrast to cell nuclei using UV-PARS and high resolution, achieved using $266 \mathrm{~nm}$ light. In our method, deep learning is used strictly for style transformation of contrast found in the input images, and does not need to infer the presence of nuclei from negative contrast. Our input images to the CycleGAN algorithm already show close concordance with true H\&E images, but the CycleGAN algorithm helps achieve a level of realism to enable our virtual histology method to offer preferred quality over FSA. Our reflection-mode approach is suitable for imaging unstained thick tissues within a few minutes, which is critical for intraoperative applications.

Alternative approaches for intraoperative imaging include white-light surgical microscopy, fluorescence-guided surgery, and other virtual histology technologies. White-light surgical microscopy alone generally does not provide adequate tumor discrimination ${ }^{40}$. Surgical fluorescence imaging with dyes that are preferential to tumor tissues have shown promise, but sensitivity and specificity is still lacking, potentially as a result of biodistribution issues, background signal, and insufficient spatial resolution. For example, Ottoliono-Perry et al. showed only $55.6 \%$ positive predictive value for tumor identification, imaging a breadloafed lumpectomy specimen outside a demarcated tumor border where margin determination is critical ${ }^{41}$.

As noted in the introduction, our approach offers some advantages over other virtual histology approaches. Methods which require staining or optical clearing procedures usually require an additional few minutes which can be eliminated in our label-free approach. Our rapid $4.2 \mathrm{~s} / \mathrm{mm}^{2}$ acquisition time for the fast-scanning mode is significantly faster than scan times reported for SRS microscopy $\left(25 \mathrm{~s} / \mathrm{mm}^{2}\right)^{28}$, recent PAM imaging systems $\left(36 \mathrm{~s} / \mathrm{mm}^{2}\right)^{24}$ or previous UV-PARS work ${ }^{6-8,13}$. Scan time could additionally be improved several-fold in future embodiments using faster repetition rate lasers and resonant galvanometer mirrors or polygon scanning mirrors. High-NA brightfield microscopes currently used by pathologists typically offer $\sim 300 \mathrm{~nm}$ resolution. Our $390 \mathrm{~nm}$ lateral resolution approaches this with our high-resolution scanning mode and resolution can be improved in future work for further discrimination of intra-nuclear structures like nucleoli.

It is worth noting some of the potential limitations of the current CycleGAN virtual staining model. Though the network was not directly presented with any examples of larger-scale histological features at low magnification during training, given the limited $125 \mu \mathrm{m} \times 125 \mu \mathrm{m}$ field of view in each training image for maximal resolution, in practice this did not appear to 

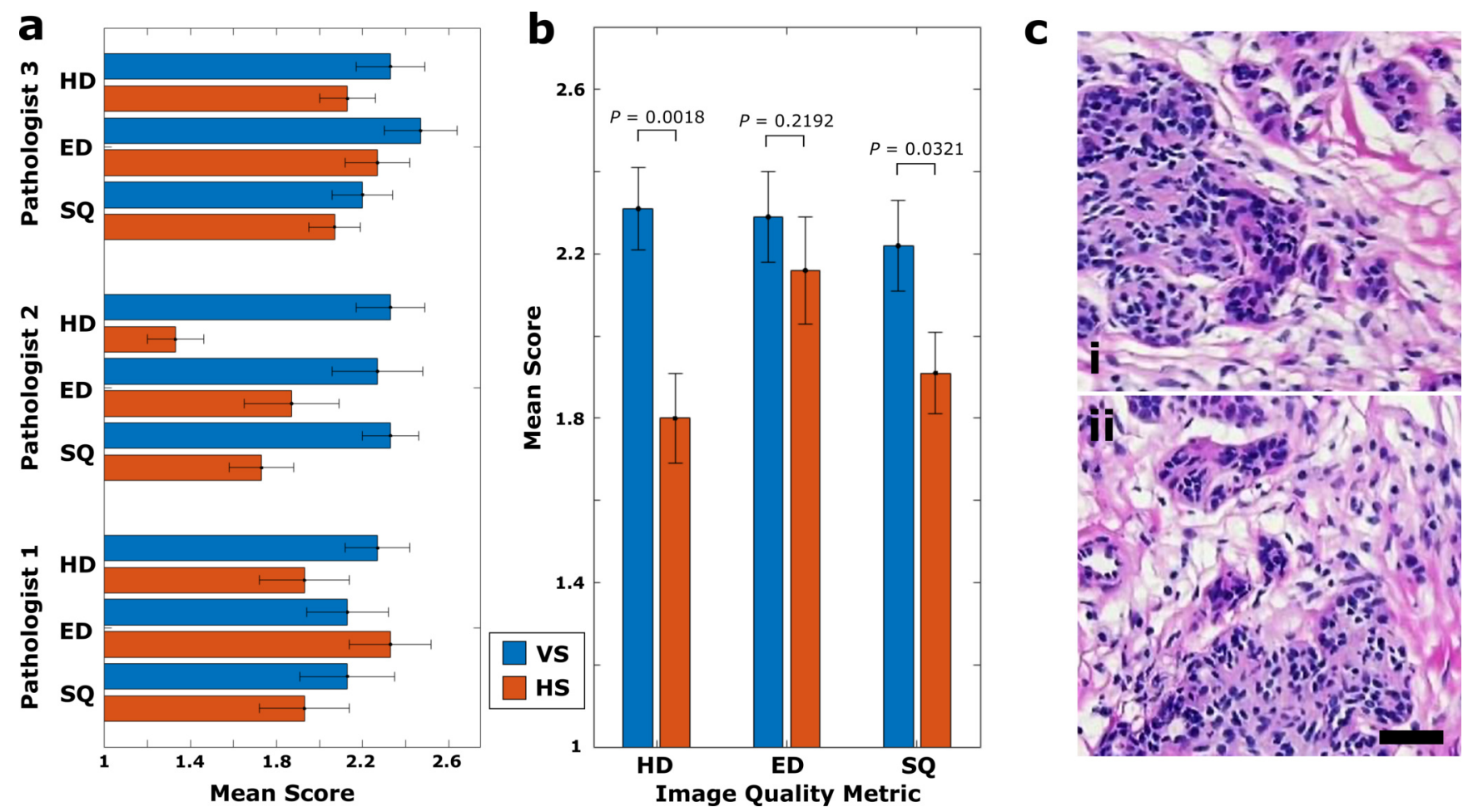

Figure 8. Summarized blinded pathologist survey results. a) Graph of the mean scores over the sample of all images provided by each pathologist for each of the image quality metrics. b) Mean results for each histological method and image quality metric using the inter-pathologist average image scores. Results are reported as mean \pm SEM. SEM, standard error of the mean; VS, deep learning-enabled virtual H\&E sample; HS, frozen section H\&E-stained histological sample; HD, hematoxylin detail; ED, eosin detail; SQ: overall stain quality. c) Representative frozen section H\&E-stained breast tissue brightfield microscopy. Scale bar: $50 \mu \mathrm{m}$. 
affect its ability to translate such features in previously unseen test images. The $125 \mu \mathrm{m}$ x $125 \mu \mathrm{m}$ training image field of view was more than sufficient to include high-magnification cytogical details in training examples, which appeared to generalize well to representing histological architecture at lower magnifications. One notable drawback of the mouse model training is the significant presence of red blood cells infiltrating the tissue in an inextricable manner throughout the training data set of true H\&E-stained images. The excitation wavelength of the UV-PARS system used in this work should not provide positive contrast to these anucleate cells, though their extensive presence in the training data results in their appearance in some translated virtual stain images. However, this effect does not appear to significantly corrupt results and a visible-wavelength excitation beam could be added to achieve simultaneous hemoglobin contrast similar to previous work ${ }^{42,43}$.

Drawbacks of FSA have been well-documented. Frozen section artifacts show abnormalities seen microscopically that do not directly reflect an abnormality in the tissue and can make interpretation difficult ${ }^{3}$. For example, ice crystals can enlarge or introduce variability in nuclear size, and can cause hole formation mimicking the appearance of intracellular vacuoles or fat. Air drying artifacts can result in indistinct cell borders and smudged chromatin, while crush artifacts can also obscure histological details, hindering accurate evaluation. Moreover, shatter artifacts can occur if the cryostat temperature is not optimal or calcifications are present, where the tissue will appear similar to Venetian blinds. Adipose tissue in particular can be challenging to cut for frozen section analysis, often leading to tissue fragmentation and folding, or an increase in thickness. Given the technical limitations in quality and diagnostic utility, several studies have found false negative rates as high as 36\% for frozen sections compared to permanent FFPE sections in certain procedures such as radical prostatectomy and breast-conserving surgery ${ }^{4-46}$. In many cancer types, concordance between frozen sections and permanent sections is high, but some cancer types such as melanoma show poor agreement ${ }^{47}$.

Our virtual histology approach may offer an alternative to FSA and provide advantages for imaging large tissue areas without the artifacts associated with freezing. Future work needs to rigorously establish the diagnostic concordance and accuracy of our virtual histology approach compared with frozen section and permanent section analysis. Currently our setup is built on an optical table in a laboratory. A cart-based system should be realized in future work to be integrated into the operative workflow.

In conclusion, we developed a high-resolution combined UV photoacoustic remote sensing and UV scattering microscope which, integrated with a CycleGAN, was able to produce virtual histology images with high quality and histological realism. A blinded pathologist study found that the nuclear detail and overall stain quality of our virtually-stained images were preferred over H\&E-stained frozen sections. Given the fast scan times, high-resolution, and pathologist preference over frozen sections, our approach offers considerable promise for future intraoperative applications.

\section{Methods}

\section{Optical imaging system}

A diagram of the optical setup used for simultaneous UV-PARS and UV scattering imaging is found in Figure 1 of the supplementary information. In the second harmonic generation subsystem, a $20-600 \mathrm{kHz}$ pulse repetition rate linearly-polarized $532 \mathrm{~nm}$ fiber laser (GLP-10, IPG Photonics) is focused through a 5 x 5 x $6 \mathrm{~mm}$ nonlinear cesium lithium borate (CLBO) crystal to generate $266 \mathrm{~nm}$ excitation light. Residual $532 \mathrm{~nm}$ light is removed from the generated $266 \mathrm{~nm}$ beam via a dispersive prism and passed into a beam dump. The $266 \mathrm{~nm}$ beam width is then expanded using a Galilean beam expander to match the objective entrance pupil. A half-wave plate is used to rotate the polarization of the $266 \mathrm{~nm}$ beam to be maximally transmissive through a polarizing beam splitter cube. The transmitted beam then passes through a quarter-wave plate converting linear into circular polarization. The circularly-polarized UV beam is then combined with an interrogation beam via a harmonic beam splitter, and both beams are co-focused onto the sample using a reflective objective. Back-scattered circularly-polarized pulsed UV light is then redirected via the quarter-wave plate and polarizing beam splitter cube onto a photodiode (PDA10A, Thorlabs). By utilizing a custom sample-and-hold peak detector circuit, the photodiode measurement of the narrow UV pulse can be held and adequately sampled by the digital acquisition card. This back-scattered signal which utilizes the existing excitation wavelength for the UV-PARS subsystem is responsible for eosin-like contrast. A continuous wave (CW) $1319 \mathrm{~nm}$ center wavelength linearly-polarized superluminescent diode (SLD1018PXL, Thorlabs) acts as the interrogation source following a similar polarization-based redirection as described previously. By co-focusing both the interrogation and excitation beams, photoacoustic-induced reflectivity modulations can be detected using the AC-coupled output of a $75 \mathrm{MHz}$ balanced photodiode (PDB420C-AC, Thorlabs) measuring back-scattered interrogation light intensity. This signal is further filtered using an in-line $20 \mathrm{kHz}$ high-pass filter (KR Electronics) to remove scanning noise. This modulated CW interrogation back-scattered signal provides hematoxylin-like contrast. The system is capable of imaging in two separate modes, a slower high-resolution 0.5 NA mode (LMM40X-UVV, Thorlabs), and a rapid lower resolution 0.3 NA mode (LMM15X-UVV, Thorlabs) with a longer working distance. The high-resolution mode utilizes stage scanning, and the fast-scanning mode utilizes constant velocity stage scanning in one axis combined with high frequency galvanometer scanning in the perpendicular axis to rapidly image strips that can be stitched together in a mosaic for large field of view imaging. 


\section{Data acquisition and processing}

For the high-resolution mode, a function generator and two digital delay generators (DDG) were used in combination to synchronize the excitation laser, digital acquisition card (DAQ), peak detection reset, and stage movement. Channel 1 of the FG was used to initiate $532 \mathrm{~nm}$ excitation lasing at a fixed pulse repetition rate of $120 \mathrm{kHz}$ using a $50 \%$ duty cycle $120 \mathrm{kHz}$ frequency square wave. Channel 2, outputting the same waveform as channel 1, was then synchronized in phase to channel 1 and time-delayed to ensure synchronization in time of pulse events with DAQ (CSE8389, Gage Applied) trigger events. This channel 2 output was fed into a DDG as the external trigger input to synchronize stage movement with excitation trigger events as outlined in previous work ${ }^{48}$. As outlined in Snider et al. ${ }^{49}$ the peak detector used to sample-and-hold back-scattered UV pulsed excitation light requires resetting between detection events to ensure no leakage of signal. This was achieved by using the synchronization output of channel 1 time-delayed such that a reset event occurs sufficiently far in time relative to the acquisition window. The DAQ captured 32 pre-trigger and 64 post-trigger data points using a $125 \mathrm{MS} / \mathrm{s}$ sampling rate to ensure the full PARS modulation and peak-detected UV back-scattered signals were detected. The maxima of these temporal signals were each associated with a single spatial location to generate both absorption and scattering contrast images. For the hybrid optomechanical fast-scanning mode, a function generator was used to control galvanometer scanning and controller feedback was fed as input into the DAQ to determine spatial locations associated with each signal, this approach is outlined in more detail in previous work ${ }^{14}$.

In order to combine hematoxylin-like absorption contrast UV-PARS images with eosin-like scattering contrast images a pseudo-colored image fusion algorithm was used. This algorithm uses a reference FFPE H\&E-stained brightfield microscopy image along with a blind source stain separation algorithm to generate independent hematoxylin and eosin colormaps that are applied to the UV-PARS and UV scattering images, respectively ${ }^{33,34}$. A composite of these two pseudo-colored images is then obtained using alpha blending with joint scaling of intensity values. This pseudo-coloring image fusion algorithm generates composite virtual images with good contrast between hematoxylin and eosin features that is then well-suited to CycleGAN input. An overview of the process flow of these steps is illustrated in Fig. 2a.

\section{Cycle-consistent generative adversarial network}

The CycleGAN architecture used for translating UV-PARS images into realistic histological images was adopted from Zhu et al. ${ }^{29}$. As shown in Fig. $2 \mathrm{~b}$, the CycleGAN model consisted of the generators $G$ and $F$, paired with discriminators $D_{Y}$ and $D_{X}$, respectively. The generators are trained to learn the translational mappings $G: X \rightarrow \widehat{Y}$ and $F: Y \rightarrow \widehat{X}$, where $X$ is an input data domain of UV-PARS images and $Y$ is an input data domain of true H\&E-stained brightfield histology images. Here, $\widehat{X}$ and $\widehat{Y}$ denote domains of translated output images, which converge toward indistinguishable statistical distributions from the corresponding $X$ and $Y$ data domains under optimized generators. Adversarial training of each generator/discriminator pair was performed using least squares loss functions $\mathscr{L}_{a d v}$ to compare candidate translated output images with input data (Fig. $2 b$ ).

The cycle consistency constraint necessary for unsupervised training on unpaired input data was enforced by forward and backward cycle consistency loss functions $\mathscr{L}_{c y c}$. These functions compute the L1 norm of the difference of an input image $x$ and its reconstruction $F(G(x))$ following a round-trip cycle of the generative networks in the forward case, or similarly compare an input $y$ with the reconstruction $G(F(y))$ in the backward case. The total loss function $\mathscr{L}_{\text {tot }}$ for the CycleGAN model used the following weighting for the individual terms:

$$
\mathscr{L}_{\text {tot }}=\mathscr{L}_{a d v}\left(G, D_{Y}\right)+\mathscr{L}_{a d v}\left(F, D_{X}\right)+\lambda \mathscr{L}_{c y c}(G, F) .
$$

A heavy weighting of the cycle consistency loss, $\lambda=150$, was tuned by experimental validation to ensure the tissue morphology of the input UV-PARS images is preserved under the translation, with the output strictly transforming the style into a realistic histological image. The identity loss term described for some applications in Zhu et al. ${ }^{29}$ was not utilized in this work, as the preservation of color composition between input and output is contradictory to the goal of our translation, where color modification is a critical degree of freedom for the network to optimize in rendering an accurate, high fidelity virtual stain.

The generative and discriminative networks of the CycleGAN are outlined in Fig. 2c. The generative networks are based on a ResNet architecture consisting of an encoder path for downsampling input images to an abstract latent space representation, nine residual blocks each with two convolutional layers for learning the translation between image domains, and a decoder using fractionally-strided convolutions to construct output images. Each convolution used reflection padding and was followed by instance normalization and a ReLU activation. The discriminator networks used a 70x70 PatchGAN architecture. This network maps $512 \times 512$ input images to a $32 \times 32$ output array, where each element represents a classification that can be traced through the series of convolutions to overlapping $70 \times 70$ receptive fields in the input image. The output array is averaged to determine the single scalar classification value for the entire input image. In this network, a leaky ReLU activation with a slope of 0.2 was used in all layers, and the first layer omitted the instance normalization. The discriminator parameters were updated based on a stored buffer of 50 generated images rather than only the latest generated example, as suggested by Zhu et al. to reduce model oscillation ${ }^{29}$. 


\section{Network training}

The training sets for true H\&E-stained brightfield microscopy of breast and prostate tissues were generated by cropping 1000 unique $125 \mu \mathrm{m}$ x $125 \mu \mathrm{m}$ images from whole-slide digital pathology scans (Aperio). The breast tissue scans were obtained at a 400x magnification, while the prostate tissues were scanned at 200x magnification. The breast training set contained equal proportions of benign tissues, and malignant tissues obtained from tumor resection cases signed-out by a pathologist with an invasive carcinoma diagnosis. This was done to ensure the network was presented with examples of both benign and malignant histology, and mitigate potential bias in the translation function output. The UV-PARS image training set also contained 1000 images with a $125 \mu \mathrm{m} \times 125 \mu \mathrm{m}$ regions with data augmentation consisting of rotations and reflections used to enlarge the data set of unique images. For a fixed grid of 512x512 pixels, there is an inherent trade-off in field of view and sampling resolution for each training image, without modifying the network to accept larger images which would drastically increase complexity in terms of learnable parameters, memory requirements and training time. In this case, relatively small training images were selected to allow for fine sampling, taking advantage of the high-resolution 400x digital pathology scans. However, the fully-convolutional nature of the network allows arbitrarily large images sampled at the same resolution to be translated following training.

For training the mouse tissue translation models, 512x512 pixel grids over a $125 \mu \mathrm{m}$ x $125 \mu \mathrm{m}$ field of view were again used for each image. The liver tissue training set consisted of 200 each of UV-PARS images and true H\&E-stained brightfield images. The kidney tissue training set contained 300 images of each type. Given the reduced amount of training examples in these cases, a transfer learning approach was employed. While the human breast and prostate translation models were trained from scratch, the mouse tissue translation models used the fully-trained human breast tissue model as a starting point. While these stained tissue images differ considerably in style, they contain similar morphological features, which we hypothesize should aid the progression of the model training.

In each case, the CycleGAN model was trained using the Adam optimization algorithm with a batch size of 1 . Training persisted for 100 epochs at a learning rate of 0.0002, which was decayed linearly to zero for a further 100 epochs of training. Training the breast and prostate tissue translation models from scratch required $\sim 55$ hours using GPU resources from Google Colab Pro. Training the mouse liver and kidney models by transfer learning with smaller training sets required $\sim 10.8$ hours and $\sim 16.3$ hours, respectively. Following training, only the optimized $G$ generative model is necessary for subsequent image translation of new UV-PARS images to realistic virtual histology, which can be performed in seconds for images $\sim 50 \mathrm{MB}$ in size.

\section{Tissue acquisition and preparation}

Formalin-fixed, breadloafed human breast tumor lumpectomy and radical prostatectomy tissue specimens were obtained from breast and prostate cancer patients, after pathology cases were closed and tissues were otherwise flagged for disposal as per approved ethics [HREBA (Cancer)/HREBA.CC- 20-0145]. Specimens representative of benign breast histology were obtained from a reduction mammoplasty procedure, where the tissue would have otherwise been discarded. Patient details pertaining to excised tumor specimens were anonymized from both research staff and pathologists involved in this study. Tissue specimens were paraffin-embedded and sectioned into $4 \mu \mathrm{m}$ thin sections, with adjacent sections being H\&E-stained for morphological comparison where possible. Sections imaged by UV-PARS were de-paraffinated and re-hydrated prior to imaging. Murine tissues were obtained in accordance with protocols approved by the University of Alberta Animal Care and Use Committee. Fresh murine kidney and liver specimens were immersed in a phosphate-buffered saline solution immediately after dissection from a Swiss Webster mouse (Crl:CFW(SW), Charles River Laboratories) following isofluorane-induced euthanasia. Freshly-resected tissues were imaged using UV-PARS within 30 minutes, prior to formalin fixation.

\section{Blind comparison of virtual histology and frozen sections}

A set of $n=15$ frozen section H\&E-stained brightfield microscopy images of human lumpectomy specimens obtained from a tissue bank (OriGene), and $n=15$ CycleGAN UV-PARS virtual images of human breast specimens obtained from benign tissues and tumor lumpectomy procedures were compiled to form a randomized blinded set of breast histology images. These images were then presented to three anatomical pathologists who were asked to rank each image based on hematoxylin detail (HD), eosin detail (ED), and overall stain quality (SQ) on a 1-4 evaluation scale, similar to the study reported by Rivenson et al. ${ }^{35}$. The numerical scale defined scores as follows: 1 , unacceptable; 2 , acceptable; 3 , very good; and 4, perfect. The results of this blinded evaluation of virtual and frozen H\&E stained breast tissue sections are tabulated in Table 1 of the supplementary information. To evaluate prospective statistical significance of the hypothesis that virtual images produced image quality that was preferred over frozen sections by pathologists, a right-tailed Wilcoxon rank sum test was performed, which is generally considered as the nonparametric version of a two-sample t-test. This test is appropriate for comparing independent samples that may not be normally-distributed, and allows the significance of differences in population medians to be evaluated, where the distributions have approximately equal variances. This assumption appears to be reasonable in our study when sample variances are used as an estimate of population variances. The test was performed for each image quality metric, using the 
exact version of the test statistic computation. Moreover, tested image scores were taken as a mean over pathologist ratings, which in part reduces the effect of interobserver variability, and avoids the problem of computing p-values where ties exist in the exact Wilcoxon rank sum test.

\section{References}

1. Orosco, R. K. et al. Positive surgical margins in the 10 most common solid cancers. Sci. reports 8, 1-9 (2018).

2. DeSantis, C. E. et al. Cancer treatment and survivorship statistics, 2014. CA: a cancer journal for clinicians 64, 252-271 (2014).

3. Lester, S. C. \& Hicks, D. G. Diagnostic Pathology: Breast, E-Book (Elsevier Health Sciences, 2021).

4. Hajireza, P., Shi, W., Bell, K., Paproski, R. J. \& Zemp, R. J. Non-interferometric photoacoustic remote sensing microscopy. Light. Sci. \& Appl. 6, e16278-e16278 (2017).

5. Reza, P. H., Bell, K., Shi, W., Shapiro, J. \& Zemp, R. J. Deep non-contact photoacoustic initial pressure imaging. Optica 5, 814-820 (2018).

6. Haven, N. J., Bell, K. L., Kedarisetti, P., Lewis, J. D. \& Zemp, R. J. Ultraviolet photoacoustic remote sensing microscopy. Opt. letters 44, 3586-3589 (2019).

7. Haven, N. J., Kedarisetti, P., Restall, B. S. \& Zemp, R. J. Reflective objective-based ultraviolet photoacoustic remote sensing virtual histopathology. Opt. Lett. 45, 535-538 (2020).

8. Abbasi, S. et al. All-optical reflection-mode microscopic histology of unstained human tissues. Sci. reports 9, 1-11 (2019).

9. Restall, B. S. et al. Virtual hematoxylin and eosin histopathology using simultaneous photoacoustic remote sensing and scattering microscopy. Opt. Express 29, 13864-13875 (2021).

10. Ecclestone, B., Dinakaran, D. \& Reza, P. H. Single acquisition label-free histology-like imaging with dual-contrast photoacoustic remote sensing microscopy. J. Biomed. Opt. 26, 056007 (2021).

11. Bell, K. et al. Reflection-mode virtual histology using photoacoustic remote sensing microscopy. Sci. reports 10, 1-13 (2020).

12. Kedarisetti, P. et al. F-mode ultraviolet photoacoustic remote sensing for label-free virtual h\&e histopathology using a single excitation wavelength. Opt. Lett. 46, 3500-3503 (2021).

13. Haven, N. J. et al. Virtual histopathology with ultraviolet scattering and photoacoustic remote sensing microscopy. Opt. Lett. 46, 5153-5156 (2021).

14. Restall, B. S. et al. Fast hybrid optomechanical scanning photoacoustic remote sensing microscopy for virtual histology. Biomed. Opt. Express 13, 39-47 (2022).

15. Olson, E., Levene, M. J. \& Torres, R. Multiphoton microscopy with clearing for three dimensional histology of kidney biopsies. Biomed. optics express 7, 3089-3096 (2016).

16. Ragazzi, M. et al. Fluorescence confocal microscopy for pathologists. Mod. Pathol. 27, 460-471 (2014).

17. Glaser, A. K. et al. Light-sheet microscopy for slide-free non-destructive pathology of large clinical specimens. Nat. biomedical engineering 1, 1-10 (2017).

18. Fereidouni, F. et al. Microscopy with ultraviolet surface excitation for rapid slide-free histology. Nat. biomedical engineering 1, 957-966 (2017).

19. Yao, D.-K., Maslov, K., Shung, K. K., Zhou, Q. \& Wang, L. V. In vivo label-free photoacoustic microscopy of cell nuclei by excitation of dna and rna. Opt. letters 35, 4139-4141 (2010).

20. Wong, T. T. et al. Fast label-free multilayered histology-like imaging of human breast cancer by photoacoustic microscopy. Sci. advances 3, e1602168 (2017).

21. Wong, T. T. et al. Label-free automated three-dimensional imaging of whole organs by microtomy-assisted photoacoustic microscopy. Nat. communications 8, 1-8 (2017).

22. Imai, T. et al. High-throughput ultraviolet photoacoustic microscopy with multifocal excitation. J. biomedical optics $\mathbf{2 3}$, 036007 (2018).

23. Kim, H., Baik, J. W., Jeon, S., Kim, J. Y. \& Kim, C. Paexm: label-free hyper-resolution photoacoustic expansion microscopy. Opt. Lett. 45, 6755-6758 (2020). 
24. Li, X., Kang, L., Zhang, Y. \& Wong, T. T. High-speed label-free ultraviolet photoacoustic microscopy for histology-like imaging of unprocessed biological tissues. Opt. Lett. 45, 5401-5404 (2020).

25. Kang, L., Li, X., Zhang, Y. \& Wong, T. T. Deep learning enables ultraviolet photoacoustic microscopy based histological imaging with near real-time virtual staining. Photoacoustics 25, 100308 (2022).

26. Sun, Y. et al. Real-time three-dimensional histology-like imaging by label-free nonlinear optical microscopy. Quant. Imaging Medicine Surg. 10, 2177 (2020).

27. Orringer, D. A. et al. Rapid intraoperative histology of unprocessed surgical specimens via fibre-laser-based stimulated raman scattering microscopy. Nat. biomedical engineering 1, 1-13 (2017).

28. Hollon, T. C. et al. Near real-time intraoperative brain tumor diagnosis using stimulated raman histology and deep neural networks. Nat. medicine 26, 52-58 (2020).

29. Zhu, J.-Y., Park, T., Isola, P. \& Efros, A. A. Unpaired image-to-image translation using cycle-consistent adversarial networks. In Proceedings of the IEEE international conference on computer vision, 2223-2232 (2017).

30. Goodfellow, I. et al. Generative adversarial nets. Adv. neural information processing systems 27 (2014).

31. Mirza, M. \& Osindero, S. Conditional generative adversarial nets. arXiv preprint arXiv:1411.1784 (2014).

32. Isola, P., Zhu, J.-Y., Zhou, T. \& Efros, A. A. Image-to-image translation with conditional adversarial networks. In Proceedings of the IEEE conference on computer vision and pattern recognition, 1125-1134 (2017).

33. Li, X. \& Plataniotis, K. N. Circular mixture modeling of color distribution for blind stain separation in pathology images. IEEE journal biomedical health informatics 21, 150-161 (2015).

34. Li, X. \& Plataniotis, K. N. A complete color normalization approach to histopathology images using color cues computed from saturation-weighted statistics. IEEE Transactions on Biomed. Eng. 62, 1862-1873 (2015).

35. Rivenson, Y. et al. Virtual histological staining of unlabelled tissue-autofluorescence images via deep learning. Nat. biomedical engineering 3, 466-477 (2019).

36. Rivenson, Y. et al. Phasestain: the digital staining of label-free quantitative phase microscopy images using deep learning. Light. Sci. \& Appl. 8, 1-11 (2019).

37. Chen, Z., Yu, W., Wong, I. H. \& Wong, T. T. Deep-learning-assisted microscopy with ultraviolet surface excitation for rapid slide-free histological imaging. Biomed. optics express 12, 5920-5938 (2021).

38. Winetraub, Y. et al. Oct2hist: Non-invasive virtual biopsy using optical coherence tomography. medRxiv (2021).

39. Li, J. et al. Biopsy-free in vivo virtual histology of skin using deep learning. Light. Sci. \& Appl. 10, 1-22 (2021).

40. Ma, L. \& Fei, B. Comprehensive review of surgical microscopes: technology development and medical applications. $J$. biomedical optics 26, 010901 (2021).

41. Ottolino-Perry, K. et al. Intraoperative fluorescence imaging with aminolevulinic acid detects grossly occult breast cancer: a phase ii randomized controlled trial. Breast Cancer Res. 23, 1-20 (2021).

42. Restall, B. S., Haven, N. J., Kedarisetti, P. \& Zemp, R. J. In vivo combined virtual histology and vascular imaging with dual-wavelength photoacoustic remote sensing microscopy. OSA Continuит 3, 2680-2689 (2020).

43. Abbasi, S. et al. Chromophore selective multi-wavelength photoacoustic remote sensing of unstained human tissues. Biomed. Opt. Express 10, 5461-5469 (2019).

44. Tsuboi, T. et al. Is intraoperative frozen section analysis an efficient way to reduce positive surgical margins? Urology 66, 1287-1291 (2005).

45. Cendán, J. C., Coco, D. \& Copeland III, E. M. Accuracy of intraoperative frozen-section analysis of breast cancer lumpectomy-bed margins. J. Am. Coll. Surg. 201, 194-198 (2005).

46. Cheng, L., Al-Kaisi, N. K., Liu, A. Y. \& Gordon, N. H. The results of intraoperative consultations in 181 ductal carcinomas in situ of the breast. Cancer: Interdiscip. Int. J. Am. Cancer Soc. 80, 75-79 (1997).

47. Prieto, V. G. et al. Are en face frozen sections accurate for diagnosing margin status in melanocytic lesions? Am. journal clinical pathology 120, 203-208 (2003).

48. Martell, M. T., Haven, N. J. \& Zemp, R. J. Fiber-based photoacoustic remote sensing microscopy and spectral-domain optical coherence tomography with a dual-function 1050-nm interrogation source. J. Biomed. Opt. 26, 066502 (2021).

49. Snider, L., Bell, K., Hajireza, P. \& Zemp, R. J. Toward wide-field high-speed photoacoustic remote sensing microscopy. In Photons Plus Ultrasound: Imaging and Sensing 2018, vol. 10494, 143-150 (SPIE, 2018). 


\section{Acknowledgements}

The authors are grateful for assistance with histology preparations from Shalawny Miller. The peak detection circuit used was originally designed by Logan Snider. The authors also thank Andrey Gorbunov for providing representative benign breast tissue samples. This work received funding from the Canadian Institutes of Health Research (PS 168936) and the Natural Sciences and Engineering Research Council of Canada (2018-05788).

\section{Author contributions statement}

R.J.Z, and X.L. conceived the experiments and supervised the research. M.T.M., N.J.M.H., and E.A.M. generated training data, and optimized and validated the machine learning methods. N.J.M.H., M.T.M., B.S.R. built the optical system and conducted the imaging experiments. M.T.M., N.J.M.H., B.S.R., and B.D.C. developed hardware and software methods for scanning and image reconstruction. M.T.M., N.J.M.H., and R.J.Z. developed the blinded study and analyzed data. B.A.A., J.D., and R.M. performed diagnosis and participated in the blinded study. L.P. and N.G. assisted with requisition of the breast and prostate tissues. J.D., N.G., M.T.M., N.J.M.H., and R.J.Z. contributed to human ethics approvals. M.T.M, N.J.M.H., and R.J.Z. prepared the manuscript. All authors reviewed and edited the manuscript.

\section{Competing interests}

RJZ is a founder and shareholder of illumiSonics Inc., which, however, did not support this work. RJZ is founder, shareholder and director of CliniSonix Inc., which, however, did not support this work.

\section{Data availability}

Relevant data and code will be made available upon reasonable request. 


\section{Supplementary Files}

This is a list of supplementary files associated with this preprint. Click to download.

- SupplementaryInformation.pdf 